\title{
XI.
}

Aus der Universitätspoliklinik für Nervenkranke und dem hirnanatomischen Laboratorium in Zürich (Professor von Monakow).

\section{Ueber einen Fall von transitorischer reiner Worttanbheit.}

\section{Von \\ Dr. Otto Veraguth}

in Zürich.

Die Erfordernisse zur Feststellung und scharfen Umgrenzung einer Aphasieform sind:

1. die Reinheit des klinischen Symptomemomplexes,

2. ein Sectionsbefund mit der minimalsten Veränderung, die eben ausreichte, um die betreffende Störung - und nur diese hervorzurufen.

Soweit die sogenannte subcorticale sensorische Aphasie in Frage kommt, sind diese beiden Postulate in keinem der wenigen Fälle erfüllt, in denen dieses Krankheitsbild beschrieben worden ist. Ueber die klinisch reinen Fälle liegen keine Sectionsberichte vor, während andererseits diejenigen, welche post mortem untersucht worden sind, entweder ron vorneherein nicht reine Worttaubheiten gewesen oder nicht bis zum Lebensende frei von Beimengungen anderweitiger Sprachstörungen geblieben waren.

Die Berechtigung, den folgenden Fall zu veröffentlichen, liegt darin, dass er diese Lücke wenigstens einigermassen ausfüllen wird.

Ich mache daher gerne Gebrauch von der liebenswürdigen Erlaubniss meines Chefs, Herrn Professor von Monakow, nachfolgend die Krankengeschichte des Patienten $R$. aus der Universitätspoliklinik für Nervenkranke in Zürich zu publiciren.

\section{Krankengeschichte.}

Peter R., 42 Jahre alt, früher Maschinenarbeiter, stammt aus gesunder Familie. Seine Fran und 5 Kinder leben und sind gesund, das jüngste ist 10 Monate alt. - Mit 19 Jahren machte Patient einen Typhus durch. Seither will er zeitweise an Kurzathmigkeit gelitten haben. Ueber andere Krankheiten wird von $R$., trotz mehrmaligen Fragens, nichts berichtet; insbesondere ist für Lues in der Anamnese kein Anhaltspunkt aufzuspüren. Dagegen giebt er unumwunden zu, früher Alkoholiker gewesen zu sein: er habe jahrelang Morgens Schnaps und den Tag über Wein und Bier getrunken. Seit 6 Jahren aber "sei er Abstinent".

Deutsche Zeitschr. f. Nervenheilkunde. XVII. Bd. 
Anfang September 1897 nun erlitt R. einen Unfall. Er wurde von der Handhabe eines rotirenden Schwungrades derart über die linke Stirnhä]fte getroffen, dass er hinfiel und ungefähr $1 / 2$ Stnnde (?) bewusstlos liegen blieb. Heimtransportirt und zu Bette gelegt, erwachte er mit Kopfweh und Tosen in den Ohren. Er konnte aber sofort reden, hören, Worte verstehen, sehen, sich ungehindert bewegen. Es bestanden keine sichtbaren Blutungen. 14 Tage lang hütete er das Bett. Dann blieb er 3 Wochen lang noch unthätig zu Hause; erst in der zweiten Octoberwoche war er im Stande, es wieder mit einiger leichterer Arbeit zu versuchen.

Seit dem Unfall, so gab Patient bei der ersten Untersuchung (18. October 1897) an, leide er an Kopfschmerzen und an Drtuckgefühl an einer Stelle am Rückgrat. Der Schlaf sei schlecht; der Appetit ordentlich; nie habe er Brechreiz verspürt. Stuhlgang regelmässig.

Es falle ihm auf, dass er viel schlechter sehe als vor dem Unfall. Dies zeige sich besonders beim Lesen; demn nach kurzem Versuch verzerrten sich die Linien and die Worte verschwämmen. Geistige Anstrengung veranlasse Steigerung der Kopfschmerzen, auch scheine ihm sein Gedächtniss abgenommen zu haben. Anfallsweise werde ihm schwindlig, so dass er sich halten mü̈sse, um nicht umzufallen.

Status im October 1897. Mässig kräftiger Mann, von ordentlichem Ernährungszustand; hat weisse Haare; sieht überhaupt viel älter aus, als er ist. Der Schädel ist an einer 5frankstückgrossen Stelle $3 \mathrm{~cm}$ über dem linken Ohr auf Beklopfen schmerzhaft. Keine Narben am Kopf oder im Gesicht. Stark geschlängelte Temporalarterien. Facialisinnervation beiderseits normal. - Die rechte Pupille ist bedeutend weiter, als die linke, reagirt träger als diese. Angenbewegungen ungehindert; bei starker Blickwendung nach links wird jedoch ein Doppelbild angegeben. Das Gesichtsfeld zeigt concentrische Einschränkung beiderseits, namentlich für Roth. Augenhintergrund normal. Grosser Druck wird gut gelesen; kleinerer (Snellen III) nur einige Zeilen weit, dann erklärt Patient, plötzlich niehts mehr deutlich zu sehen. - Zunge gerade herausgestreckt. Keine Geschmacks- oder Geruchsstörungen. - Am Hals und an den Armen nichts Besonderes; kein Tremor der gespreizten Finger. Untere Extremitäten ohne abnormen Befund. Gang sicher, ohne Schwanken, kein objectives Zeichen von Schwindel. - Auf der linken Lunge leichter Katarrh des Oberlappens. Sonstiger Befund der Thoraxorgane normal. Am Abdomen fällt ausser einem linksseitigen Leistenbruch nichts Besonderes auf. - Die Reflexe sind normal, abgesehen vom Patellarphänomen, das links deutlich erhöht ist. Die Prüfnng der Sensibilität ergiebt, dass Knopf und Spitze der Stecknadel uiberall richtig unterschieden werden; es besteht keine Differenz in der Intensität der Berührungsempfindung zwischen rechts und links; Schmerz- und Temperaturempfindung unverändert. Dagegen scheint der stereognostische Sinn der Hände etwas herabgesetzt: Patient taxirt mit der linken und rechten Hand ein 20 Cent.stück für ein 5 Cent.stück; ein 2 Francstück für ein 1 Francstïck. - Händedruck rechts 26 , links 26 Kilo. - Urin eiweiss- und zuckerfrei.

Dieser Zustand änderte sich im Verlauf von zwei Monaten insofern, als Patient nach einiger Zeit im Stande war, wieder anhaltender zu lesen und sein Kopfweh ihn allmählich verliess. Auch waren nach Kurzem keine Doppelbilder mehr bei extremer Blickwendung nach links vorhanden, und es verlor sich die Störung des stereognostischen Sinnes. 
Um die Jahreswende 1897/1898, also 10 Wochen nach der ersten Untersuchung, klagt R. nach einem längeren Spaziergang über Unsicherheit im linken Fuss. Hat das Gefühl, derselbe sei geschwollen. Fr kann sich nicht mehr auf die linke Fussspitze heben (rechts kann er es). Die Sensibilitätsprüfung der unteren Extremitäten ergiebt keine Anomalien. Patellarreflex beiderseits vorhanden, links stark erhöht. Sohlenreflexe beiderseits gleich. Wade rechts 32 , links $29 \mathrm{~cm}$. Oberschenkel über der Patella rechts $351 / 2$, links $33 \mathrm{~cm}$; Mitte zwischen Trochanter und Patella rechts $461 / 2$, links $451 / 2 \mathrm{~cm}$. Die vier kleinen Zehen des linken Fusses sind stark extendirt. Flexion des Oberschenkels geschieht links weniger kräftig, als rechts. Beim Marschiren wird der linke Fuss in Hackenstellung aufgesetzt. Die elektrische Prüfung ergiebt leichte Herabsetzung der Erregbarkeit des Peroneus, keine EaR.

Zu gleicher Zeit klagte R. über Dyspnoë nnd Husten. Systolisches Blasen über der Mitralis. Puls 80, irregulär. Urin eiweiss- und zuckerfrei.

Im Verlanf des Januar und Februar 1898 blieb die Neuritis im linken Bein - um eine solche handelte es sich offenbar - ungefähr gleich. Dagegen entwickelte sich in dieser Zeit eine Laryngitis und Bronchitis. Unter geeigneter Behandlung traten diese Beschwerden jedoch bald zurück.

Da - am 28. Februar 1898 - überraschte uns R. durch ein gänzlich unerwartetes Symptom. Mit seinem Namen im Wartezimmer aufgerufen, reagirt er nicht. Zwei-, dreimaliges, lauteres Anrufen erregt seine Aufmerksamkeit nicht. Er muss vom Nach barn durch Anstossen mit dem Ellbogen anf den wartenden Arzt aufmerksam gemacht werden. Sodann tritt er ruhig und ohne Gangstörung in das Sprechzimmer ein. Auf die Frage: "Nun, R., wie geht es Ihnen denn heute?" antwortet er mit einem verlegenen Lachen: „Ich verstehe Sie nicht. Ich verstehe überhaupt nicht mehr, was man zu mir sagt. Aber ich höre Alles ganz gut. Ich höre auch, wenn man zu mir spricht. Aber wenn mir meine Frau etwas sagt, so ist es ein Kauderwälsch, das ich nicht verstehe. Und mit den andern Leuten geht es mir auch so." Durch ein Zeichen aufgefordert, sich zu setzen, folgt er dem Wink. Ein in diesem Augenblick auf der Strasse ertönendes Horn veranlasst ihn zur sofortigen Aussage: "Ich höre zum Beispiel ganz gut, dass jetzt gerade ein Horn auf der Strasse geblasen wird." Ohne dass er es sieht. wird der Inductionsapparat in Thätigkeit gesetzt. Er versteht das Experiment sofort, indem er bemerkt: „Auch die Maschine höre ich genau, wie früher." Es wird ihm eine Uhr von hinten her vor das $O h r$ gehalten, ohne ihn zu berühren. Er constatirt ohne Zögern, dass er auch die Uhr ticken höre (b is zu einer Entfernung von $1 / 2$ Meter beiderseits). Auf Fragen antwortet er nur, wenn er sieht, dass gesprochen wird, nicht, wenn man ihn von hinten her anspricht. Seine Antwort ist aber beständig dieselbe: "Ich verstehe kein Wort von dem, was Sie sagen; es ist mir, wie ein Gebrumm im Ohr, so ein Sausen." A uch wenn man mit erhoboner Stimme, scharf articulirend und langsam Worte des täglichen Verkehrs ihm zuruft, versteht er 
gar nichts. Seine spontane Rede aber ist fliessend, absolut ohne Wortverwechslung oder Verstïmmelung der Worte. Er giebt spontan an, dass er oft die Zeitung lese. Er könne aber nicht behalten, was er lese. Es werden ihm Druckproben vorgehalten; er liest sie fliessend, ohne die geringste Paraphasie. - Nach seiner Angabe bestehe dieser Zustand seit einigen Tagen. Er habe eine Reise nach Bern gemacht und dort, um sich zu stärken, wieder einmal Wein getrunken. Die schriftlich ihm mitgetheilte Mahnung: „Lassen Sie sofort alles Wein- und Biertrinken wieder wegi", kann er, wenn auch etwas langsamer, als die Druckschriftproben, lesen. Sie erregt eine etwas inadäquate Heiterkeit. Er verspricht, ihr za folgen. Tags darauf giebt er aber wieder za. neuerdings spanischen Wein getrunken zu haben. Er glaube, heute einzelne Worte zu verstehen. Die Untersuchung des äusseren Ohres ergiebt keine Anomalien des Trommelfelles. Er höre beständiges Sausen, namentlich im linken Ohr, schwächer im rechten.

Am 4. März 1898 trat Morgens ohne besondere Veranlas. sung starkes Nasenbluten auf. Von diesem Moment an sei ihm bedeutend leichter geworden and er habe alsbald auch Alles wieder verstanden, was man zu ihm gesprochen. Nur sei noch das Sausen im linken Ohr übrig geblieben; rechts aber sei es verschwunden.

Am 7. März 1898 berichtet $R$. in Uebereinstimmung mit seinen früheren Aussagen, dass er die vorigen Tage hindurch, während er nichts verstand, doch jedes Geränsch gehört habe. Ja, er behauptet sogar, feiner gehört zu haben, als sonst; doch habe er kein einziges gesprochenes Wort verstanden. Er giebt spontan an, es habe ihm jede Anrede ,wie ein e fremde Sprache geklungen". Es sei ihm schon vor einiger Zeit (und zwar am 20. November 1897, wie sich dies in einem Actenstück feststellen lässt) einmal aufgefallen, dass er auf einem Bureau den Beamten, der mit ihm sprach, zll seinem Erstaunen plötzlich absolut nicht verstanden habe. Er habe dann, aus Verlegenheit, mit den Schuhen sich zu schaffen gemacht, und glaube, nach diesem tiefen Bücken wieder leichter verstanden zu haben.

Kurze Zeit darauf reiste Patient, ohne seine Familie ins Einverständniss zu setzen, wieder nach Bern und wurde dort im Inselspital längere Zeit beobachtet. Dem Brief eines befreundeten Collegen, nnter dessen Anfsicht R. stand, entnehme ich, dass daselbst ein Klappenfehler, starke Herzarhythmie, die sich oft bis zum Delirium cordis steigerte, ferner Emphysem und Asthma constatirt wurde. Durch geeignete Behandlung seien diese Symptome wesentlich gebessert worden. Nie habe sich eine Spur von einer Sprachstörung mehr gezeigt; auch dann nicht, als man ihm, experimenti causa, ausnahmsweise Wein verabreicht habe. Die Neuritis des linken Beines sei ungefähr gleich geblieben.

Am 28. Juni 1898 stellte R. sich wieder in der Poliklinik. Er sei jetzt imner so ängstlich. Sein Gedächtniss sei noch immer schlecht. Er weiss das Datum seines Unfalls nicht anzugeben. Der Schädel ist nicht mehr percussionsempfindlich. Die rechte Pupille ist noch immer weiter als die linke; Pupillenspiel beiderseits normal. Herzdämpfung $2 \mathrm{~cm}$ rechts vom Sternalrand, $3 \mathrm{~cm}$ links von der Mamilla. Stürmische, irreguläre Herz- 
action. Radialispuls sehr schwach, 130. Lungengrenzen nach unten verbreitert, über der ganzen Ausdehnung der Lunge feuchtes Rasseln. Wadenumfang rechts $31 \frac{1}{2}$, links $29 \mathrm{~cm}$.

Bis Ende Februar 1899 zeigte der inzwischen noch oft untersuchte Patient ungefähr den eben geschilderten Status, mit kleinen Schwankungen des Zustandes der Lungen und des Herzens. Auch seine subjectiven Beschwerden blieben im Ganzen dieselben. Zur Arbeitsfähigkeit brachte er es nie mehr. Von einem Wiederauftreten der Störung des Sprachverständnisses oder der Sprache überhaupt jedoch zeigte sich nie mehr auch nur eine Spur.

Hier mag beigefügt werden, dass $R$. für seinen Unfall seinerzeit $z u$ seiner Zufriedenheit entschädigt worden ist, und zwar lange vor dem Auftreten der oben geschilderten sehweren Störungen; so dass schon dadurch, wie übrigens durch den ganzen objectiven Befund zur Genïge Simulation der fraglichen Symptome ausgeschlossen ist.

Ende Februar 1899 erkrankte R. unter den Symptomen einer croupösen Pneumonie des rechten Unterlappens. Nach 5 Tagen Krisis. Am 2. März 1899 mehrere Schüttelfröste; nachherige hohe Temperatnren (bis $40^{\circ}$ ), mit morgendlichen Remissionen. Rasseln auf dem rechten Ober- und Mittellappen. Herzarhythmie. Nach einigen Tagen wird der Auswurf putrid. Am 11. März 1899 plötzliches Erblinden des rechten Auges. Pupille jetzt mittelweit (die früher immer weit gewesen), zeigt grünlichen Reflex. Das kranke Auge hat nur noch Lichtschein. - Znnehmende Schwäche, häufge Dyspnoë. 12. März Abends unwillkürliche Bewegungen im linken Arm (nach Angabe der Frau); am anderen Morgen lassen sich dieselben nicht mehr constatiren; Patient kann den Arm frei bewegen. 14. März Morgens 6 Uhr Exitus letalis unter Erscheinungen des Collapses.

14. März Morgens $9 \mathrm{Uhr}$ Section. Starke Abmagerung. Icterische Verfärbung der Haut. Fettpolster sehr gering. Musculatur spärlich, von guter Farbe. Beim Eröffnen des Thorax liegen die Lungen in starker Ausdehnung vor. Der Herzbeutel ist aber zum Theil unbedeckt. Beim Anschneiden desselben entleeren sich ca. $200 \mathrm{ccm}$ gelblich-grüner, trüber Flüssigkeit. Das parietale und viscerale Blatt des Pericards ist durch Auflagerungen getrübt und vielfach verdickt. Aus den Vorhöfen entleert sich Cruor und Speckhaut. Herz vergrössert; beide Kammern etwas weiter, als normal, Tricuspidal- und Pulmonalklappen ohne besonderen Befund. Mitralis kaum für einen Bleistift durchgängig, mit stark verdicktem, verkalktem Rand. Auf einem Zipfel sitzt ein Thrombus, der ausgedehnte Nekrose zeigt; bei leichtem Betupfen zerfällt er in Fetzen. Aortenklappen und Aorta ohne Besonderheit, Anfang der letzteren nicht verkalkt. Musculatur des Herzens beiderseits stark verdickt, brann verfärbt. Rechte Lunge an der Spitze adhärent. Beim Loslösen derselben entleeren sich ca. $50 \mathrm{ccm}$ Eiter in die - vorher leere - Plenrahöhle. Pleura ausser an der Anheftungsstelle intact. Im Unterlappen der Lunge kein Luftgehalt, mässiger Blutgehalt. Emphysematös erweitert. Im Oberlappen inselförmige Verdichtungen, wo das Organ nicht Iufthaltig ist. Bronchien mit Eiter gefüllt. Linke Lunge nicht adhärent, Pleura glatt, sie zeigt anf der Aussenseite des Oberlappens unter der Pleura eine streifenförmige Eiteransammlung. Auf dem Durchschnitt ist sie gut luft-, wenig bluthaltig, an der eben genannten Stelle verdichtet. Leber vergrössert, zeigt das Bild der Staunng. 
Milz und Nieren ohne Besonderheiten. L. Bulbus oculi zeigt Abhebung der Retina durch eine frische Blutung hinter dieselbe. - Schädeldach aussen und innen ohne Besonderheiten. Die Schädelknochen sind dünn, zeigen wenig Diploë. Dura der Oberfläche nirgends verwachsen. Im Sinus longitudinalis Crnor und Speckhaut. Pia der Oberfläche beiderseits über dem Scheitel stark ödematös, ihre Gefässe reichlich gefüllt. Ueber dem Mantelrand der rechten Hemisphäre in der Gegend des Paracentralläppchens liegt unter der Pia ein frischer Eiterherd in der Ausdehnung eines 1-Francstückes. Consistenz der Rinde in dieser Gegend etwas weicher. Starke Pacchioni'sche Granulationen. Dura der Schädelbasis glatt, feucht, glänzend. Schädelbasis ohne Fracturspuren. Pia der Basis zart. Opticus und Oculomotorius von fibrösen Strängen begleitet. Arteriae vertebr., basil., carotis int. und Fossae Silv. mässig erweitert und geschlängelt; Intima glatt, die Gefässe sind mit geronnenem, nirgends an der Gefässwand adhärentem Blut gefüllt.

Die linke Hemisphäre zeigt im hinteren Theil der dritten Stirnwindung (in der Pars opercularis) bedeutende Atrophie. Dieser Theil der Windung fehlt fast ganz und ist durch seröse Flüssigkeit ersetzt. Ebenso zeigt die Mitte der 1. Temporalwindung einen Defect nach der 1. Temporalfurchezu, an dessen Stelle sich ebenfalls zwischen Gehirn und Häuten seröse Flüssigkeit findet. Auch im Uebrigen ist die 1. Temporalwindung schmal; die 1 . Temporalfurche $3 \mathrm{~cm}$ tief. Central-und Interparietalfurche, sowie der Sulcus frontalis II sind beträchtlich tiefer, als normal, und mit seröser Flüssigkeit gefüllt. Die 2. Frontalfurche communicirt mit dem Ramus ascendens der Fiss. Silvii. Auf der rechten Hemisphäre fällt die Schmalheit der 1. Temporalwindung auf. Sie ist unten eingefasst von einer $2 \mathrm{~cm}$ tiefen Temporalfurche. Hinterhorn erweitert gegenüber der anderen Hemisphäre (Occipitalspitze bis Spitze des Hinterhorns links $51 / 2$, rechts $4 \mathrm{~cm}$ ). Ebenso Unterhorn erweitert. Auch links ist der Seitenventrikel weiter, als normal. Ependym nicht granulirt. Die weisse Substanz des Grosshirns ist in toto reducirt und zeigt zahlreiche, gleichmässig vertheilte Blutpuncte. Auf dem Durchschnitt sind die oben beschriebenen Windungen kammartig zugespitzt. Die Sehhügel zeigen makroskopisch keine Veränderung. Das Corpus geniculatum internum erscheint links etwas grösser, als rechts. Facialis normal, Acusticus beiderseits etwas schmal (?), von normaler Farbe.

Grosshirnmantel und Hirnstamm, nach Meynert getrennt, wurden in Formol-Müller gehärtet, nachdem einzelne Partien der atrophischen Windungen herausgeschnitten und behufs Färbung nach Nissl in Alkohol gelegt worden waren. Die grossen Präparate wurden sodann im Gudden'schen Mikrotom in Serien geschnitten und einzelne Schnitte aus den wichtigsten Gegenden mit Carmin gefärbt.

Das Ergebniss dieser genaueren Durchsuchung war folgendes: Die schon oben als atrophisch geschilderten Gyri zeigen ihre Reduction, ihre kammförmig zugespitzte Gestalt und ihre Begrenzung durch breite und tiefe Furchen auch auf den Durchschnitten. Die Rinde erscheint in diesen Gebieten namentlich, in geringerem Grade aber überall etwas schmäler, als in normalen Controlpräparaten. Auch die Markmasse ist in toto 
reducirt. Von einem Herd in Rinde oder Mark ist keine Spur zu finden. Die Gefässe sind mässig erweitert; ihre Wand nicht abnorm gestaltet; es lassen sich zwar an einzelnen Stellen per diapedesin ausgetretene Lymphocyten längs einiger Capillaren erkennen; doch zeigen sich nirgends erhebliche Blutanstritte. Die Gefässwände sind nicht verändert. Die Nisslpräparate geben keine Auskunft darüber, auf Rechnung welcher Elemente die Verschmälerung der Rinde zu setzen sei. Insbesondere sind die kleinen Pyramidenzellen in Schnitten aus der 1. Temporalwindung links wohl erhalten. Einige unter ihnen zeigen Randstellung des Kernes. In den grossen Pyramiden der Centralwindungen starke gelbliche Pigmentbildung. - Acusticuskerne, untere Schleife, hinterer Zweihügel, Corpus geniculatum internum zeigen unter dem Mikroskop keine wesentlichen Veränderungen. Ebensowenig die anderen Theile des Hirnstammes. Die oben signalisirte Verschiedenheit der beiden Corpora geniculata interna erwies sich als auf ungleicher Ueberlagerung durch die benachbarten Organe beruhend. Die Gefässe dieser Gehirntheile sind etwas weit; doch halten sich die Dimensionen innerhalb normaler Grenzen. Veränderungen der Gefässwände sind nicht nachzuweisen.

Das Wesentliche in der Geschichte dieses Falles, die, da es sich die längste Zeit, und namentlich während der interessantesten Periode, um ambulante, poliklinische Beobachtung handelte, nothgedrungen etwas skizzenhaft ausfallen musste, lässt sich etwa folgendermassen zusammenfassen:

Ein hereditär nicht belasteter, vielleicht durch einen früher durchgemachten Typhus, wahrscheinlich durch langjährigen Alkoholmissbrauch in seinem Nervensystem geschädigter Mann erleidet ein Schädeltrauma. Von den unmittelbaren Folgen desselben, die auf keine localisirte Hirnläsion schliessen lassen, erholt er sich alsbald. Doch bleiben ihm mit einiger Hartnäckigkeit Störungen des Gesichtssinns: Pupillendifferenz, concentrische Gesichtsfeldeinschränkung für Farben und eine anfangs ausgesprochene, allmählich abklingende Dyslexie. Von weiteren accentuirten Erscheinungen seitens des Nervensystems kann neben einer vorübergehenden leichten Störung des stereognostischen Sinnes, neben dem Auftreten von Schwindel, Ohrenșausen und Kopfweh nichts von Belang constatirt werden. Auch diese Symptome klingen langsam ab. Doch bleibt von Anfang an eine entschiedene Herabsetzung der psychischen Persönlichkeit des Patienten bestehen, ohne dass sich aber neben etwelcher Gedächtnissschwäche und der Unfähigkeit, zu arbeiten, bestimmte psychische Krankheitszeichen feststellen liessen. Im Anschluss an eine körperliche Ueberanstrengung stellt sich eine schnell auftretende Parese des linken Beines mit geringer Atrophie ein - periphere Neuritis. Zu gleicher Zeit wird - nachdem bei der Eintrittsuntersuchung von Seiten dieses Organes keine Anomalien bemerkt worden waren, am Herzen ein 
Mitralklappenfehler mit Compensationsstörungen constatirt. Ca. 1/2 Jahr nach dem Unfall, und unleugbar im Anschluss an alkoholische Excesse nach angeblicher mehrjähriger Abstinenz, tritt eine wohlumschriebene Sprachstörung ein, die nach kurzer Dauer gänzlich verschwindet und nie mehr auftritt. Im letzten 1/2 Jahr beherrscht die Herzaffection das Krankheitsbild; sie manifestirt sich jetzt in Dilatatio cordis, in starker Arhythmie und in consecutiven Erscheinungen seitens der Lungen. Tod durch Endocarditis mit Embolien in die Lungen und andere Organe.

Sectionsbefund: Pericarditis, Endocarditis ulcerosa valv. mitralis, putride Bronchopneumonie, frische eitrige Embolie unter der Pia des Mantelrandes der rechten Hirnhemisphäre. Stauungsleber. Postretinales Hämatom im rechten Bulbus. Allgemeine Atrophie des Grosshirns, besonders der Rinde und des Marks der 1. Temperalwindung beider Hemisphären und von der Pars opercularis der dritten Stirnwindung links. Keine Herdläsionen. -

Im Vordergrund des Interesses steht die Sprachstörung, an der R. während einer kurzen Zeit litt. Es handelt sich hier um eine transitorische "Worttaubheit" oder, nach der häufig noch gebräuch lichen älteren Nomenclatur, "subcorticale sensorische Aphasie", und zwar um eine durch keine Beimengung anderweitiger aphasischer Störungen verwischte, also um ein klinisch reines Bild.

Folgende Analyse wird diese Diagnose rechtfertigen.

1. Die Aphasie war im Falle R. eine sensorische. Denn es lagen keinerlej. Störungen seitens der expressiven Sprachsphäre vor.

2. Die sensorische Aphasie war eine sogenannte "subcorticale" d. h., soweit das klinische Bild in Frage kommt. Denn alle - oder doch alle wesentlichen - Erfordernisse zu dieser Diagnose waren vorhanden: R. sprach während der Dauer der Affection ebenso geläufig, wie vorund nachher, und - was betont werden muss -, ohne dass eine Spur von Paraphasie hätte bemerkt werden können. Das laute Vorlesen ging bei gedruckter Schriftprobe geläufig und ohne Paraphasie vor sich. Das Schriftverständniss war nun freilich nach Angabe des Patienten dadurch getrubt, dass er einmal nach Kurzem „,in den Augen“ ermüdete, d. h. dass sich ihm die Buchstaben nach dem Lesen längerer Abschnitte verzerrten; zweitens aber durch eine Vergesslichkeit und Unfähigkeit, sich zu concentriren, die wohl als Theilerscheinung der ganzen Herabsetzung der psychischen Persönlichkeit anzusprechen ist, nicht aber als specielle receptive Sprachstörung. Was die in diesen Fällen minder wichtige willkürliche Schrift und das Copiren von Vorlagen anbelangt, muss zugegeben werden, dass diese Versuche bei der kurzen Dauer der Affection der poliklinischen Untersuchungsart zum 
Opfer fielen. Es liegt aber kein Grund vor zur Annahme, dass sich Störungen in jenen Beziehungen ergeben hätten. Wichtiger aber ist, dass die positiven Ergebnisse der Untersuchung unanfechtbar sind. Der Patient, dessen Gehör für Klänge und Geräusche erhalten - er meinte sogar gesteigert - war, verstand kein Wort von dem, was man zu ihm sprach. Später wieder in den Besitz des Wortverständnisses gelangt, giebt er an, dass während jener Zeit jede Anrede ihm wie eine fremde Sprache geklungen habe. $\mathrm{Ob}$ er damit sagen wollte, er habe zwar wohl verstanden, dass man rede, nur nicht, was man sage, lassen wir dahingestellt. Wahrscheinlicher ist, dass er nur dann den Eindruck hatte, dass man rede, wenn er es an den Lippen des Untersuchenden sehen konnte. Jedenfalls erregte (und dies mag hier betont werden) die Klang- und Geräuschfolge gesprochener Worte als solche, d. h. als acustisches Phänomen, seine Aufmerksamkeit nicht: er reagirte auf Anrufen mit seinem Namen im Wartezimmer nicht, sondern musste durch einen Rippenstoss von seinem Nachbar auf den rufenden Arzt aufmerksam gemacht werden. Ebenso machte es auf ihn keinen Eindruck, wenn hinter seinem Rücken gesprochen wurde, während er, wenn er sah, dass man zu ihm sprach, meist mit der Betheuerung antwortete, er höre schon, aber er verstehe nichts. Man ist daher zur Annahme veranlasst, dass seine Aufmerksamkeit für Geräusche überhaupt abgestumpft war, unbeschadet der Schärfe der Perception derselben nach Erregung der Aufmerksamkeit auf anderem Wege. Doch sei hier hervorgehoben, dass seine einmal erregte Aufmerksamkeit sehr schnell nachliess, dass er auch eine gewisse stumpfe Gleichgültigeit gegenüber seiner auffallenden Ausfallserscheinung zur Schau trug. Sein Gesicht zeigte nicht den Ausdruck der Schwerhörigen, die sich eifrige Mühe geben, zu verstehen; auch nicht den der Depression, wie sie etwa bei der schweren, plötzlich eintretenden Läsion zu erwarten gewesen wäre. Wie weit bei der Würdigung dieser Thatsachen die alkoholische Intoxication mit in Betracht kommt, muss dahingestellt bleiben. - Es ist klar, dass bei dieser Unfähigkeit, Worte zu verstehen, von Nachsprechen und von Schreiben nach Dictat nicht die Rede sein konnte.

3. Die "subcorticale sensorische Aphasie" war in diesem Falle eine reine. Dies verdient, mit Nachdruck betont zu werden. Wir sind berechtigt, ihr dieses Adjectiv zu vindiciren, da sich vor Allem von Paraphasie beim willkürlichen Sprechen und Lautlesen keine Spur zeigte. Denn bekanntlich ist auch bei der viel häufiger beschriebenen, differential-diagnostisch hier allein in Frage kommenden "corticalen" sensorischen Aphasie der Verlust des Sprachverständnisses das hervorragendste Symptom. Für diese charakteristisch ist dann aber die 
Begleiterscheinung der Paraphasie: die Patienten bedienen sich beim willkürlichen Sprechen und Lautlesen öfters oder seltener, aber ausnahmslos, verdrehter Worte oder solcher, die ähnlich klingen, wie die beabsichtigten. Ueberdies ist bei „corticaler" sensorischer Aphasie auch das Schriftverständniss aufgehoben. Von Alledem nichts bei unserem Fall. Das Bild ist daher als Sprachstörung in "centripetaler" Richtung scharf umschrieben.

Weniger scharf freilich ist seine Abgrenzung "nach der Peripherie" zu. War die Störung sicher nur eine cerebrale oder spielte auch eine Läsion der peripheren Gehörorgane eine Rolle? Als Antwort auf diese Frage stehen uns nun freilich spärliche objective Daten zur Verfügung. Fine Trübung oder anderweitige Veränderung des Trommelfells lag keinerseits vor. Von einer Prüfung der Hörschärfe durch Flüstersprache und mittelst Stimmgabeln konnte angesichts des erschwerten Verkehrs mit dem Patienten die paar Mal, da er worttaub auf die Poliklinik kam, aus naheliegenden Gründen nicht die Rede sein. Eine Untersuchung des Gehörs, nachdem die sensorische Aphasie wieder spurlos verschwunden war, gab uns auch keinen Aufschluss darüber, ob während der Störung eine periphere Componente mit im Spiel war. Wir sind also auf die spontanen Angaben des Patienten während und nach dem Anfall von Aphasie angewiesen. Er hörte jedenfalls nicht schlecht: das Ticken der Uhr erkannte er in der Entfernung von $1 / 2 \mathrm{~m}$ auf beiden Ohren (nachdem durch die Untersuchung seine Aufmerksamkeit für acustische Eindrücke geschärft worden war). Wenn also die periphere Gehörsleitung und die Perception von acustischen Phänomenen eine Veränderung erlitten hatte, so kounte es sich nur um eine so geringe Verminderung des Hörvermögens handeln, dass sie jedenfalls dem einzigen Beurtheiler, auf den wir uns in diesem Fall verlassen müssen, dem Patienten selbst, nicht auffallen konnte. Darauf freilich, dass R. sogar angab, er glaube während der Worttaubheit Geräusche noch deutlicher gehört zu haben, als vor der Attacke, ist wohl kein Gewicht zu legen. Vielmehr müssen wir annehmen, dass sich auf dem Hintergrund der Worttaubheit die annähernd normal erhaltene Fähigkeit der Wahrnehmung anderer Gehörsempfindung für das subjective Urtheil des Patienten pointirter hervorhob und dadurch den Eindruck erhöhter Hörschärfe machte. Nicht ausser Acht zu lassen ist aber das Obrensausen, das den Patienten seit dem Unfall belästigte. Und zwar dürfte mit Nachdruck hervorgehoben werden, dass dasselbe bis zum Eintritt der Worttaubheit nur auf das linke Ohr beschränkt war; dass es hingegen während der Sprachstörung beiderseits auftrat und endlich mit dem schnellen Aufbören der aphasischen Erscheinung ebenso plötzlich rechts wieder verschwand. 
4. Die "subcorticale sensorische Aphasie" war im Falle R. eine transitorische, von kurzer Dauer. Das vorübergebende Erscheinen eines so auffälligen Symptomes, wie eine reine Worttaubheit es ist, verlangt in der That eine besondere Würdigung. Rufen wir uns in Erinnerung: R. gab an, schon vor dem in der Poliklinik beobachteten Zustand der Worttaubheit einmal für kurze Zeit die Leute nicht verstanden zu haben. Dann trat die krankhafte Erscheinung mit voller Kraft auf, um einige Tage ungeschwächt anzudauern; darauf, im Anschluss an heftiges Nasenbluten, verzog sich der Schleier über dem Wortverständniss wieder und seither ist bis zum Tod jede Spur von Recidiv ausgeblieben.

Dieses transitorische Auftreten und spurlose $\nabla$ erschwinden der Sprachstörung ist schon allein eine Veranlassung, den Fall R. den bisherigen in der Literatur vorkommenden gegenüber zu stellen. Vollends aufgefordert dazu wird man durch den Sectionsbefund: allgemeine geringgradige Atrophie der interessirten Grosshirnpartien, Abwesenheit eines jeglichen subcorticalen Herdes.

Die Auffassung des Wesens der reinen Worttaubheit hat seit der ersten Feststellung des klinischen Krankheitsbildes durch Lichtheim und Wernicke nicht unbedeutende $W$ andlungen durchgemacht.

Im Jahr 1885 veröffentlichte Lichtheim den ersten Fall. ${ }^{1}$ ) Es handelte sich um einen Patienten, der im Jahr 1872 einen Schlaganfall erlitten hatte, an den sich folgende Sprachstörungen anschlossen: Paraphasie beim willkürlichen Sprechen und Vorlesen, und Paragraphie. Davon blieben im Verlauf der nächsten Jahre nur" noch Spuren der Paraphasie übrig. Im Jahr 1882 zweiter apoplectischer Insult ohne begleitende Allgemeinerscheinungen; vorübergehende Facialisparese, neuerliche Sprachstörung: Patient versteht kein einziges Wort, obwohl er alle Geräusche hört. Er habe anfangs auf Lichtheim den Eindruck eines peripher Tauben gemacht, weil er auch eine geringe Aufmerksamkeit für Geräusche an den Tag gelegt und scheinbar gar nicht darauf geachtet habe, wenn man mit ihm sprach. - Fähigkeit nachzusprechen, sowie nach Dictat zu sprechen, aufgehoben. Die willkürliche Sprache dagegen ist vollkommen correct, es kann nicht die geringste Paraphasie mehr constatirt werden. Intelligenz und willkürliche Schrift völlig intact; Lautlesen und Copiren geschieht fehlerlos. - Dieser Zustand dauerte ohne Schwankung 3 Jahre lang, bis zum Tode des Patienten. Keine Section.

1) Lichtheim, „Ueber Aphasie“. Deutsches Archiv für klinische Medicin, Band 36. S. 238. 1885 . 
Im folgenden Jahre befasste sich Wernicke ${ }^{1}$ ) mit der Frage. Auch er brachte Notizen über einen Fall ähnlicher Art, der aber durch anderweitige cerebrale Störungen (epileptische Anfälle) complicirt war. Obwohl beide Forscher für ihre Befunde keine Belege durch die Section erheben konnten, wurde von ihnen die Affection mit dem topisch präjudicirenden Namen der "subcorticalen sensorischen Aphasie" benannt. Dabei gingen sie ron der Annahme aus, dass es sich um eine Erkrankung im Marklager des linken Temporallappens handeln müsse. Ihnen war also das Krankheitsbild ein Herdsymptom par excellence und eine rein cerebrale Störung der Sprachperception.

In der That schien diese Annahme eine Stütze zu erhalten in dem 1892 erschienenen Bericht über einen Sectionsbefund bei einer "subcorticalen sensorischen Aphasie", die Pick ${ }^{2}$ ) beobachtet hat. Pick's Kranker war ein Irrsinniger, der wiederholte Schlaganfälle, unter anderen auch mit linksseitiger Hemiplegie durchgemacht hatte. Das Sprachverständniss fehlte ganz. Fragen beantwortete er falsch, insofern er zwar an sich correcte, aber nicht zur Sache gehörige Bemerkungen machte. Auf Geräusche in seiner Umgebung achtete er nicht. Es bestand bei ihm thatsächlich ein geringer Grad beiderseitiger Taubheit. Er percipirte indessen leiseres Rufen, Sprechen u. s. w. Das Verständniss gehörter Melodien schien ebenfalls gestört, er konnte aber etwas Flöte spielen. Die Sprache war correct, also frei von Paraphasie. Vorgezeigte Gegenstände bezeichnete er richtig: Die Schrift war langsam, aber correct. Ebenso richtig das fliessend von statten gehende Lesen, für welches das Verständniss erhalten war. Nachsprechen und Dictatschreiben fehlte vollkommen. Der Zustand des Patienten änderte sich während der Beobachtungszeit nicht. Section: Rechts: Gyrus temp. I und ziemlich grosse Theile des Gyrus temp. II, ferner die ganze Insel und kleine umschriebene Stellen am unteren Ende der vorderen Centralwindung und in der untersten Stirnwindung sind erweicht, und zwar in Rinde und Mark. Links: Hintere Hälfte des Gyrus temp. I und des Gyrus supramarginalis erweicht.

Nun war aber dieser Fall, obwohl durch eine Section "ergänzt, in keiner Beziehung unzweideutig. Erstens war er klinisch nicht rein weder als cerebrale Störung (Complication mit einer Geisteskrankheit), noch mit Bezug auf eventuelle Mitbetheiligung peripher erkrankter Hörbahneṇ. Ueber letztere fehlen genauere Daten. Zweitens war die Zerstörung des Gehirns, welche durch die Autopsie blossgelegt wurde, eine viel zu ausgedehnte, um einen einigermassen sicheren Schluss

1) Wernicke, „Die neueren Arbeiten über Aphasie“. Fortschritte der Medicin. 1886.

2) Pick, Archiv f. Psychiatrie. Bd. XXIII. 1892. 
auf einen Herd zu erlauben, der speciell die betreffende Störung hätte hervorgerufen. Immerhin ist bemerkenswerth - und Pick macht darauf ausdrücklich aufmerksam - dass die Läsion beide Hemisphären getroffen hatte, nicht nur die linke, und dass sowohl Rinde als Mark ergriffen waren.

Die gleiche Beobachtung machte Edgren ${ }^{1}$ ), der einen weiteren Fall veröffentlichte. 34 jähriger Patient. Kopftrauma, im Anschluss daran verschiedene cerebrale Symptome. Kann Musik nicht auffassen. Er höre sie wohl, aber nicht wie gewöbnlich; er könne keine Melodie herausfinden. Versteht seine Frau nicht, wenn sie zu ihm spricht. Zwei Tage später bleibt die Worttaubheit constant und meist complet, nur ab und $\mathrm{zu}$ versteht er einzelne Worte. Er sagt, dass er höre, wenn man $\mathrm{zu} \mathrm{ihm}$ spreche, er verstehe aber nichts. Die Sprache ist meist fliessend, zuweilen aber paraphasisch; Lautlesen geht anfangs leicht und correct, nach einer Weile aber mit Paraphasie. Das Schreiben, anfangs correct, wird später öfters durch Paragraphie entstellt. Das Gehör ist beiderseits herabgesetzt. Nach einer Woche versteht er ziemlich gut, wenn man über etwas spricht, womit seine Gedanken beschäftigt sind. Auf anderen Gedankengang reagirt er erst, wenn man ihn schüttelt, bevor man ihn anredet. Später versteht er Alles, was langsam geredet wird, kann nachsprechen und Dictatschreiben. Zuletzt bleibt als Rest der Affection geringe Paraphasie und Tontaubheit. Zwei Jahre später Tod durch Purpura haemorrhagica. Section: Aeltere Erweichungsherde in der Oberfläche der Gehirnhemisphären: links im vorderen Theil der Fossa Sylvii eine Einsenkung, die die vordern zwei Drittel der 1. Temporalwindung und die vordere Hälfte des Gyr. temp. II umfasst. Am Uebergang zwischen hinterem und mittlerem Drittel des Gyr. temp. I ist die oberflächliche Schicht sklerotisch und mit der Pia verwachsen. Unter diesem sklerotischen Theil und $1 \mathrm{~cm}$ weiter nach hinten geht die Erweichung auch in das Mark des Gyr. temp. I. Die umliegenden Theile der Gehirnrinde sind gesund. Rechts: Um den Ramus horizontalis foss. Sylvii ähnliche Defecte. In der Rinde sind zerstört die äussere und die obere Oberfläche der hinteren Hälfte des Gyrus temp. I und entsprechend der untere Rand des Gyrus supramarginalis. Die Zerstörung ging im Boden der Fossa Sylvii in die weisse Substanz über und war nach vorne und nach hinten ausgedehnter, als in der Rinde. - Also auch hier Zerstörung von Rinde und Mark in beiden Hemisphären. Nicht die Klarheit des Symptomencomplexes und Unzweideutigkeit des anatomischen Befundes verleihen dem Fall seine

1) Edgren, Zeitschrift f, Nervenheilkunde. Bd. VI. 
Bedeutung - es bestanden ja neben der Worttaubheit manche Parastörungen der expressiven Sprachsphäre und die bei der Autopsie blossgelegten Zerstörungen weisser und grauer Grosshirnsubstanz waren weit ausgedehnte - vielmehr ist es der Umstand, dass die Störung: eine nur kurze Zeit andauernde war und dass das Wortverständniss wieder vollständig sich einstellte, was angesichts des beigefügten Sectionsbefundes (subcorticale Herde beiderseits!) hervorgehoben zu werden verdient. Dies und nebenbei die Thatsache der ähnlichen Aetiologie eines vorhergegangenen Kopftraumas machen Edgren's Fall zu einem für uns besonders interessanten.

So wenig die Frage nach dem Sitz dex Erkrankung durch diese Fälle erklärt war, um so complicirter wurde sie durch die Erwägungen, die nun andere Forscher in dieser Sache geltend machten.

Schon Pick ${ }^{1}$ ) hatte in seinem Fall auf die Rolle hingewiesen, die eventuell eine periphere Gehörsstörung bei der „subcorticalen sensorischen Aphasie" spielen könnte, ohne jedoch genauere Daten über das Hörvermögen des Patienten anzugeben.

Zu gleicher Zeit schrieb Bleuler ${ }^{2}$ ) seine Arbeit "Zur Auffassung der subcorticalen Aphasien", worin er die Idee geltend machte, dass das Wortverständniss aufgehoben werden könne durch eine allgemeine Gehörsstörung, ohne dass die Perception anderer Schallqualitäten erheblich verändert sei. Er ging dabei von der Beobachtung aus, dass man im Nebenzimmer befindliche Personen zwar so gut sprechen höre, wie man ziemlich leise Geräusche dortselbst vernehme; dass man auch die Stimme des Sprechenden kenne, aber dennoch die Worte nur. höchst unvollständig oder gar nicht verstehe. Es wären also die Möglichkeiten vorhanden, dass physikalische, ausser dem Ohr liegende Hindernisse das Wortverständniss erschweren. Ebensogut könnten aber auch Ohrenkrankheiten oder drittens Affectionen des corticalen Gehörcentrums das Symptom verschulden, indem sie im letzteren Fall den geregelten Ablauf der Klangbilder verlangsamten und dadurch ein Ineinanderfliesssen der verschiedenen Worttheile bewirkten.

Soweit die Rolle der infranucleären Theile der Hörbahn dabei in Frage kommt, ist Bleuler's Ansicht namentlich von Freund ${ }^{3}$ ) eifrig verfochten worden, der in seiner Arbeit „Labyrinthtaubheit and Sprachtaubheit" den fraglichen Symptomencomplex in zwei Fällen (von denen der eine identisch ist mit dem oben erwähnten Fall Wernicke's) auf Läsion des Labyrinthes zurückführt. Fr vergleicht diese zwei Aphasiker mit Taubstummen, deren Gehör nicht vollständig ge-

1) 1. c.

2) Neurolog. Centralblatt. Bd, 18. 1892.

3) Freund, Labyrinthtaubheit und Sprachtaubheit. Wiesbaden 1895. 
schwunden ist, und findet eine grosse Aehnlichkeit im Verhalten der zwei Krankheitsformen, der Unterschied des Sprachverständnisses sei nur ein gradueller. - Seine Auseinandersetzung gipfelt in dem Satz, dass es verschiedene Arten von Sprachtaubheit gebe. „Es handelt sich um ein Symptom von Seiten des acustischen Apparates, welches keinen absoluten topischen Werth besitzt. Die veranlassende Läsion ist nicht an eine bestimmte Stelle gebunden; sie kann im Gehirn, im Acusticusstamm oder im Labyrinth des inneren Ohres, ja sogar unter Umständen im Mittelohr localisirt sein und den gleichen Functionsansfall veranlassen. Seine locale Färbung exhält das Symptom der Sprachtaubheit erst durch die gleichzeitig vorhandenen anderweitigen Symptome." Leider wird der Werth der bedeutenden Arbeit Freund's beeinträchtigt durch das Fehlen pathologisch-anatomischer Belege für seine Deductionen. Auch ist der erste seiner zwei Fälle (Wernicke's Fall Hendschel) jedenfalls cerebral erkrankt (s. o.).

Unter dem Zeichen der Bleuler-Freund'schen Ansicht von der Bedeutung der peripheren Gehörsstörungen für die Worttaubheit steht die bezüglich des Gehörs genauere Untersuchung im Fall Ziehl's. ${ }^{1}$ ) Ein 75 jähriger Mann erkrankt plötzlich, ohne Allgemeinerscheinungen, an Worttaubheit und Paraphasie. Durch den Insult wird das Verständniss für Worte zerstört, obwohl Patient hört, dass gesprochen wird. Er hört die Uhr ticken. Gehörprüfung nach Rinne und Weber ergeben normalen Befund. Er erkennt einzeln ausgesprochene

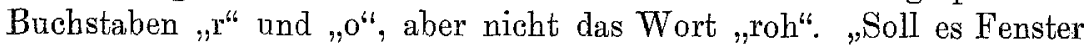
heissen"? frägt er. Dabei Paraphasie leichteren Grades. Er spricht gelegentlich von "Don Wan" statt „Don Juan", "weich" statt „reich", kann nicht nachsprechen. Einzelne Buchstaben ist er zwar im Stand, nachzuahmen, auch einzelne Onomatopoëtica, z. B. „wau, wau", aber kein einziges Wort. Das Dictatschreiben ist unmöglich. Bei der willkürlichen Schrift besteht Paragraphie. Das Lautlesen und Bezeichnen von Gegenständen geschieht mit gelegentlicher Paraphasie. Dieser Zustand bleibt unverändert bis zum Tod des Patienten. Keine Section. - Das Wesentliche an dieser Krankengeschichte liegt wohl in dem negativen Befund der Gehörsprüfung. Andererseits aber war der Fall kein rein subcorticaler (Beimengung von Parastörung), und es fehlte die Autopsie.

Hatte bis dahin das Studium der „subcorticalen sensorischen Aphasie" nur theoretische Vermuthungen zu Tage gefördert, so be-

1) Ziehl, „Ueber einen Fall von Worttaubheit und das Lichtheim'sche Krankheitsbild der subcorticalen sensorischen Aphasie". Deutsche Zeitschrift für Nervenheilkunde. VIII. 1896. 
deutete der Fall, den Sérieux ${ }^{1}$ ) klinisch beobachtet und Déjerine ${ }^{2}$ ) anatomisch untersucht hat, einen wesentlichen Fortschritt in der Erkenntniss dieser Affection.

Im Fall Sérieux-Déjerine handelte es sich um eine 55jährige Frau, die vom Jahr 1887-1893 gradatim das Verständniss für gesprochene Worte verlor, ohne dass die Hörschärfe sich verminderte. Auch konnte sie Melodien nicht mehr erkennen, noch nach Dictat schreiben; dagegen sprach sie, schrieb sie spontan, las und copirte und hatte ihre volle Intelligenz bewahrt. Bemerkenswerth ist, dass ihr längere Zeit die Fähigkeit erhalten blieb, einzelne gebräuchliche Worte und Silben zu verstehen und dadurch mit mehr oder weniger Glück die Frage des Untersuchenden zu errathen. Z. B. „En quelle année sommes-nous"? Antwort: "Somme? quelle somme"? oder: „La mémoire est-elle bonne"? "armoire? Je n'ai pas d'armoire. J'entends bien tout ce que rous me dites: Je ne suis pas sourde. Mais je ne comprends pas ..." - Einige Monate vor dem Tode traten paragraphische und paraphasische Störungen, sowie allgemeine Verminderung der Intelligenz hinzu, so dass sie zuletzt überhaupt nicht mehr schreiben, noch sich ausdrücken konnte. - Bei der Autopsie zeigten sich die Schläfenlappen beiderseits en masse atrophirt. Ihre Atrophie war symmetrisch und jeder Lappen war beinahe um die Hälfte vermindert. Sie präsentirten eine sehr dentliche Mikrogyrie und die Insel lag unbedeckt. Die Schläfenwindungen hatten ihre Form im Allgemeinen beibehalten, waren aber um die Hälfte reducirt und waren „d'une apparence lamellaire". Die Consistenz der Rinde war vermehrt. Die Pia zeigte Adhärenzen. Die mikroskopische Untersuchung des in Serien zerlegten Gehirns ergab, dass es sich um eine Poliencephalitis chronica handelte, mit beinahe vollständiger Atrophie der kleinen Pyramidenzellen und Schwund der Tangentialfasern. Die Gefässe zeigten verdickte Wände. Nirgends war ein Herd im Mark nachzuweisen, wohl aber eine merkliche Verminderung der. Türckschen Bündel (im Vergleich zu normalen Präparaten).

Mit diesem Sectionsbefund war wohl die alte Lichtheim-Wernicke'sche Annahme vom subcorticalen Herd gründlich widerlegt wie dies Déjerine in seiner Zusammenfassung des Resultates ausspricht: „Dans la surdité verbale pure il s'agit non pas d'une séparation $d u$ centre auditif des mots; mais bien d'un affaiblissement dans les fonctions du centre auditif commun". Nun muss freilich zugegeben werden, dass die Gehöruntersuchung in diesem Fall nicht eine exacte

1) Sérìieux, Revue de Médicine. Août. 1893.

2) Dejerine et Sérieux, „Un cas de surdité verbale pure terminé par aphasie sensorielle suivi d'autopsie." 1897. 
war (wenigstens fehlen darüber nähere Angaben) - so dass also klinisch jedenfalls nicht entschieden werden konnte, ob die Abschwächung des Hörvermögens wirklich nur anf die Perception der Worte sich bezog, ob es sich also d'un affaiblissement dans les fonctions du centre auditif handelte oder ob auch eine periphere Läsion mit im Spiele war. Auch blieb der Fall nicht bis zum Lebensende eine Surdité verbale pure, sondern verwandelte sich in eine Aphasie sensorielle („corticale sens. Aph."). Eine beträchtliche Zeit lang aber war die Worttaubheit eine reine gewesen. Und dennoch von einem subcorticalen Herd keine Spur!

Eine kürzlich erschienene Arbeit von Liepmann ${ }^{1}$ ) versucht nun aber doch, die alte Lichtheim-Wernicke'sche Anschaung aufrecht zu erhalten. Der Autor resumirt die Krankengeschichte seines Falles folgendermassen: „Ein 67 jähriger Mann verlor durch zwei offenbar cerebrale Anfälle, deren Symptome (rechtsseitige Krämpfe, rechtsseitige Facialislähmung) beide Male auf die linke Hemisphäre wiesen, das erste Mal vorübergehend, das zweite Mal dauernd jegliches Sprachverständniss, und zwar ist schon die Auffassung der Sprachelemente, d. i. der einzelnen Buchstaben, gestört. Das Gesprochene erkennt er überhaupt nur dann als Gesprochenes, wenn er das Gesicht des Sprechenden sieht oder vorher davon verständigt ist, dass man zu ihm sprechen werde. Das Hörvermögen für Töne erweist sich als vollkommen ausreichend für das Sprachverständniss. Die gesammte Tonreihe wird beiderseits percipirt, nur zeigt sich eine gleichmässige Herabsetzung der Hörschärfe beiderseits, wie sie weder für Labyrintherkrankung noch für Erkrankung des äusseren Gehörapparates charakteristisch ist. Insbesondere ist das von Bezold abgegrenzte Gebiet lückenlos vorhanden. Alle übrigen Sprachfunctionen sind intact. Einzelne Erscheinungen weisen über Sprachtaubheit hinaus" (partielle Seelentaubheit). Eine dritte Attacke mit Bewusstseinsverlust und Krämpfen führte den Tod herbei. Die Section ergab Folgendes: Frische Erweichungen im Pons, im Crus cerebelli ad pontem; in der rechten Hemisphäre die Ventrikel stark erweitert und mit Blutgerinnsel erfüllt; die Ventrikelwände intact; ,überhaupt in Mark und Rinde der rechten Hemisphäre kein Herd aufzufinden. In der linken Hemisphäre wird ein grosser frischer Blutherd aufgedeckt. Die Blutung hat eine mit Blutgerinnsel und zertrümmerter Hirnmasse gefüllte Höhle im Mark gebildet, die $2 \mathrm{~cm}$ hinter dem Stirnpol beginnt und bis $7 \frac{1}{2} \mathrm{em}$ vor den Occipitalpol

1) Liepmann, „Ein Fall von reiner Sprachtaubheit." Psychiatr. Abhandlungen, herausg. v. Wernicke. 1898.

Dentsche Zeitschr. f. Nervenheilkunde. XVII. Bd. 
reicht, bis in die Höhe der Stelle, an welcher das Hinterhorn sich vom Unterhorn trennt. Die Blutung hat den Thalamus und den Rest des Corpus striatum gänzlich von der Rinde abgesprengt. Oben ist die Höhle durch den zum Theil erhaltenen Balken begrenzt. Seine grösste Breite hat der Herd $6 \mathrm{~cm}$ hinter dem Stirnpol, wo er einen seitlichen Fortsatz in die dritte Stirnwindung sendet. Vorn ist der Nucleus caudatus und der Linsenkern fast ganz zerstört. Durch die Zerstörung der grossen Ganglien bilden Vorderhorn und Cella media mit der pathologischen Höhle einen Hohlraum. In der Inselgegend wird die Höhle kleiner; immerhin ist der grösste Theil des Corpus striatum und der inneren Kapsel, sowie Claustrum und äussere Kapsel zerstört. Von oben reicht in der Sagittalrichtung ein nach unten abgetrennter, $\check{\mathrm{c}} \mathrm{cm}$ langer Rest des Stabkranzes in die Höhle, diese in 2 Hälften theilend. Die Basis der Höhle wird in der Inselgegend nur durch eine $3 / 4 \mathrm{~cm}$ dicke Platte gebildet, welche das obere Dach des Unterhorns bildet. Der ganze, oberhalb dieser Platte gelegene Stabkranz zum Schläfenlappen ist zerstört. Der sich nach hinten verschmälernde und spitz in der den Thalamus nach hinten begrenzenden Frontalebene endigende Herd bleibt dauernd oberhalb des Unterhorns durch die etwa $4 \mathrm{~mm}$ dicke Platte, welche hinten den erhaltenen Rest des Stabkranzes enthält, vom Unterhorn getrennt. Unterhorn und Hinterhorn ausserordentlich erweitert und mit Blutgerinnsel erfüllt, aber intact."

Aus diesem Befund glaubt Liepmann schliessen zu müssen, dass die seinerzeit vorhandene Sprachstörung durch einen Herd im Marklager bedingt gewesen sei. "Leider hat die neue Blutung" (finale Attacke) „eine ungeheure Zerstörung angerichtet und auch gerade das Gebiet betroffen, in welchem der alte Herd zu suchen war, nämlich das Marklager des linken Schläfenlappens. Ein makroskopischer Nachweis des alten Herdes in der mit Blutgerinnsel und zertrümmerter Hirnsubstanz erfüllten Höhle, welche die Blutung hergestellt hatte, liess sich nicht führen. Zum Glück gestattet ein Exclusionsschluss schon vor der mikroskopischen Untersuchung unsere Annahme, dass der Herd im Stabkranz des linken Schläfenlappens innerhalb des neuen Herdes gelegen habe, zu bestätigen“... „In unserem Fall erwies sich nämlich die Rinde beider Schläfenlappen als intact. $\mathrm{Ja}$, in der rechten Hemisphäre ist überhaupt kein Herd nachzuweisen." ... „Da sich somit in den erhalten gebliebenen Theilen des Gehirns der alte Herd nicht fand, so muss er in dem zerstörten Gebiet, nämlich dem Mark der linken Hemisphäre gelegen haben. Doppelseitige Labyrintherkrankung war ja schon durch den klinischen Befund auszuschliessen. Der rechte $N$. acusticus ist schon mikroskopisch (van Gieson'sche und Markscheidenfärbung) untersucht worden and erwies sich als 
durchaus normal." - Ein Bericht über die mikroskopische Untersuchung der Rinde steht aber noch aus.

Liepmann's Schlussfolgerung von der Abhängigkeit der Sprachstörung von einem subcorticalen linksseitigen Herd ist durchaus nicht zwingend. Vorausgesetzt auch, dass seinerzeit im Gebiet des grossen, letzten Herdes ein kleinerer Herd in der linken Hemisphäre existirte, so ist dadurch noch immer nicht bewiesen, dass die Worttaubheit von einer Unterbrechung der cerebralen Hörbahn abhing. Vielmehr muss die Möglichkeit offen gelassen werden, dass ein solcher Herd indirect, durch Störung der Circulation in der Rinde des Schläfenlappens (Druckwirkung) Ursache der Aphasie war. Angenommen also, dass ein solcher Herd früher vorhanden war, so ist damit aber noch immer nicht die endgültige Lösung der Frage gegeben: Welches ist die minimale Hirnläsion, welche eben genügt, um die reine Worttaubheit - und nur diese - hervorzurufen? Mangels einer mikroskopischen Untersuchung der Rinde scheint der Fall überhaupt noch nicht spruchreif, namentlich auch rücksichtlich' der Frage, ob die rechte Hemisphäre unbetheiligt gewesen sei. - Ein besonderes Verdienst hat sich Liepmann's Arbeit erworben durch eine peinlich genaue Berücksichtigung eventueller infranucleärer Gehörsstörungen. Er erweitert den Kreis der üblichen Gehörprüfung durch die Bezold'sche Untersuchung mit der ununterbrochenen Tonreihe. - Wie weit die diagnostische Bedeutung dieser Untersuchungsmethoden für sensorische Aphasien geht, bedarf zwar wohl noch der Prüfung. Bezold ging bei der Aufstellung seines Lehrsatzes, dass für das Sprachverständniss unumgänglich nothwendig die Tonstrecke $b^{\prime}-g^{\prime \prime}$ sei, vom Studium des Gehörs bei Taubstummen mit Hörresten aus. Auch Freund wählte als Ausgangspunkt für seine Annahme von dem Sitz der Worttaubheit im Labyrinth die Analogie mit Taubstummen. Die Berechtigung dieser Zusammenstellung ist aber durch keine pathologisch-anatomischen Befunde in Labyrinthen bei sensorisch Aphasischen bis jetzt erhärtet. Wohl aber spricht im klinischen Bild ein Umstand direct gegen sie. In den meisten Krankengeschichten (Lichtheim, Ziehl, Liepmann, unser Fall, um die klinisch reinsten zu nennen) wird berichtet, dass die Worttauben acustischen Eindrücken gegenüber eine gewisse Gleichgültigkeit, einen Mangel an Aufmerksamkeit entgegenbringen, die scharf contrastiren gegen den Eifer, mit dem peripher Schwerhörige acustische Eindrücke zu erhaschen suchen, und gegen die seelische Reaction, die sich doch wohl bei plötzlich eintretender gewöhnlicher Schwerhörigkeit einzustellen pflegt. - Immerhin dürfte unterstrichen werden, dass in Liepmann's Fall die genane Untersuchung des Gehörs keine Labyrinthoder Mittelohrstörung ergab. 
Wie verhält sich nun unser Fall R. zu den bisherigen Ergebnissen der Forschung über das Wesen der reinen Worttaubheit?

I. Was das anatomische Substrat der cerebralen Störung betrifft, so beweist es für's Erste zur Evidenz, dass ein Patient eine reine Worttanbheit par excellence durchmachen kann, ohne einen subcorticalen Herd im linken Schläfenlappen zu besitzen. Darin ergänzt or den Fall Sérieux-Déjerine, mit dem er gemeinsam hat, dass die Läsion die minimalste war, die bis jetzt durch Section von worttauben Gehirnen anfgedeckt worden ist; ja noch mehr! Déjerine findet in der Rinde der 1. Temporalwindung beiderseits Schwund der kleinen Pyramidenzellen, in unseren nach Nissl behandelten Präparaten aus derselben Region waren aber Veränderungen gegenüber normalen Controlpräparaten nicht zu erkennen. Freilich artete auch bei Serieux's Kranken die Affection in eine gewöhnliche Aphasie sensorielle aus, während sie bei R. während ihres kurzen Bestandes frei von anderweitigen aphasischen Störungen blieb.

II. Ferner giebt die Beiderseitigkeit der allgemeinen Atrophie (Verschmälerung) der 1. Temporalwindungen eine starke Stütze ab für die Annahme von der Bilaterralität der nothwendigen Rindenerkrankung, die durch die Sectionen ron Pick, Edgren und Déjerine wahrscheinlich gemacht, durch Liepmann's Fall aber noch keineswegs zurückgewiesen ist.

III. Sodann wird durch die Autopsie in unserem Falle demonstrirt, dass von der Rinde an abwärts bis zum Acustieus keine mikroskopisch nachweisbaren Veränderungen der Hörbahn zu bestehen brauchen, damit die reine Worttaubheit zu Stande kommt. Nehmen wir dazu noch den Umstand, dass das während der Attacke nicht wesentlich gestörte und nach derselben sofort und vollständig leistungsfähige Gehörorgan direct gegen eine erhebliche Läsion des oder richtiger der Labyrinthe spricht, so dürfte der Fall $R$. eine Illustration sein zu dem Satz, dass die Worttaubheit im Wesentlichen eine corticale, resp. eine von der Grosshirnrinde ausgehende Störung ist.

Doch bleibt bei dieser Schlussfolgerung ein Widerspruch zwischen dem klinischen und dem anatomischen Bild zu lösen. Wie ist es möglich, dass R.: Worttaubheit transitorisch war, dass aber die Section eine Atrophie der 1. Schläfenwindung beiderseits aufwies? R. verstand demnach nach Ablauf, der Attacke das gesprochene Wort trotz bestehender Atrophie der beiderseitigen Gyri temporales I. Daraus ergiebt sich der zwingende Schluss, dass nicht einmal dieser Sectionsbefund der minimalste ist, der vorausgesetzt werden muss zum Zustandekommen der reinen Worttaubheit. Hier muss freilich wiederholt werden, dass die Nissl-Präparate keinen befriedigenden Aufschluss gaben über die 
Ursache der Windungsatrophie: ob und welche Zellen in geringer Anzahl fehlen oder verkleinert sind, ob anderweitige intracelluläre Veränderungen vorlagen, oder ob intracorticale Bahnen gelitten hatten. Sei dern, wie ihm wolle: die "subcorticale sensorische" Aphasie wird in unserem Falle durch den negativen Befund, theilweise wenigstens, in das Bereich der sogenannten functionellen Symptomencomplexe verwiesen.

Damit ist man nun freilich mit der Nachfrage nach der auslösenden Ursache der transitorischen Worttaubheit bei R. auf Erklärungsversuche zurückgewiesen. Doch bieten Krankengeschichte und Autopsie eine plansible Erklärung des Phänomens. Vergegenwärtigen wir uns, dass Patient an Sklerose der Hirnarterien litt und dass sich im Lauf der Beobachtung ein Herzfehler manifestirte. Derselbe mag vielleicht schon früher, schon seit dem Typhus vor 22 Jahren, bestanden haben. Jedenfalls aber gingen erst dem Anfall von Worttaubheit Compensationsstörungen des Herzens voraus. Durch Alkoholgenuss schädigte der Patient seinen defecten Circulationsapparat in noch höherem Grade, und dies konnte genügen, um in dem betreffenden Ast der Art. foss. Sylvii eine locale Ernährungsstörung leichterer, vorübergehender Art zu provociren, durch welche die aus anderen Gründen schon irgendwie defecte (leicht atrophische) graue Substanz der Schläfenwindungen in noch schlechtere Circulationsverhältnisse kam. Dadurch wurde die Reizschwelle der corticalen Neurone derart erhöht, dass die von der Peripherie kommenden Reize nicht mehr genügten zur Auslösung der associativen Arbeit des Wortverständnisses, während sie eben noch ausreichten für die Wahrnehmung und für das Verständniss von Geräuschen des täglichen Lebens. Auf den causalen Zusammenhang zwischen Circulations- und Sprachstörung weist auch die Thatsache, dass $R$. bei einem früheren, leichteren Anfall die Worttaubheit will zum Verschwinden gebracht haben dadurch, dass er sich bückte also jedenfalls durch eine Aenderung der Circulation im Schädel; ferner dadurch, dass ein heftiges Nasenbluten - also wieder eine starke Druckschwankung in den Gefässen des Kopfes - die Besserung des Zustandes herbeiführte. - Ein anderes Agens, das in diesem Falle vielleicht mit ebenso viel Wahrscheinlichkeit als auslösende Ursache kann angesprochen werden, ist der Alkohol, den R. - nach seiner Angabe nach 6jähriger Abstinenz - kurz vor dem Anfall zu sich genommen hatte. Die Möglichkeit ist nicht abzuweisen, dass die schon minderwerthigen (atrophischen) Schläfenwindungen für das Gift eine besondere Affinität besassen, die sich dann durch die Worttaubheit äusserte.

IV. Was die Frage nach der Mitbetheiligung des Labyrinths am 
Krankheitsbild anbelangt, ist allerdings einzuräumen, dass die Gehörprüfung in unserem Fall nicht in erschöpfender Weise vorgenommen wurde. Das attackenweise Auftreten der Worttaubheit, das rasche Einsetzen und Abklingen derselben, ferner das Fehlen jeder auffallenden Gehörsstörung im späteren Verlauf sprechen aber deutlich gegen eine Labyrintherkrankung, die zudem auch an beiden Ohren gleichzeitig und acut hätte auftreten müssen. Für eine Mitbetheiligung dieses Organs spricht in unserer Krankengeschichte nur das Auftreten von beidseitigen subjectiven Geräuschen. Doch bleibt dahingestellt, ob dieses Ohrensausen in die Labyrinthe zu localisiren oder nicht schliesslich auch centralen Ursprungs sei - möglich, dass sie mithalf, den corticalen Vorgang des Wortverständnisses zu erschweren.

Die Behauptung, dass periphere Störungen des Gehörs bei der reinen Worttaubheit eine Rolle spielen müssen, wird übrigens direct widerlegt durch die extrem genaue Gehöruntersuchung in Liepmann's Fall, wo keine oder nur sicher belanglose Hördefecte festgestellt wurden.

Soll aus dem Vorhergehenden der gegenwärtige Stand der Frage über das Wesen des besprochenen Krankheitsbildes resumirt werden, so lassen sich folgende Sätze aufstellen:

Das primäre cerebrale Substrat der reinen Worttaubheit ist nicht eine Unterbrechung der subcorticalen Hörbahn im Mark des linken Schläfenlappens; vielmehr genügt eine einfache Atrophie der ersten Schläfenwindungen wahrscheinlich beider Hemisphären - als anatomische Grundlage, um beim Hinzutreten eines secundären (circulatorischen, toxischen, functionellen) Momentes die reine Worttaubheit zu bedingen.

Das klinische Bild der reinen Worttaubheit bleibt, so wie es Lichtheim zuerst beschrieben hat, zu Recht bestehen. Die Beobachtung der Autoren bezüglich begleitender peripherer Gehörsstörungen machen es wahrscheinlich, dass diesen in gewissen Fällen eine Rolle in der Genese der Worttaubheit zukommt. Dieselbe kann aber nur eine secundäre sein.

Bezüglich der Nomenclatur dürfte die vielfach noch gebräuchliche Bezeichnung "subcorticale sensorische Aphasie" zu ersetzen sein durch die anatomisch nichts präjudicirende und den Kern der Sache treffende Benennung "Surdité verbale pure" der Franzosen, die "reine Worttaubheit". 